\title{
NASA buys into the action on Keck telescope
}

Washington. The US National Aeronautics and Space Administration (NASA) is close to signing an agreement with the California consortium that operates the Keck telescope, the largest optical instrument in the world, under which the space agency will pay onesixth of the Hawaiian observatory's construction costs in exchange for an equal proportion of viewing time.

NASA will use the two 10-metre Keck telescopes, the second of which is scheduled to be completed in 1996, for a long-term programme to search for planets around other stars. One-third of the viewing time on the Keck II telescope will be dedicated to that search.

The deal, due to be finalized within the next several weeks, calls for NASA to pay $\$ 6.8$ million this year, with equal amounts paid out over each of the next five years, to a total of about $\$ 40$ million. NASA's investment will be the only government money received by the observatory, which is financed largely through a private grant from the W. M. Keck Foundation.

The first Keck telescope began regular operations last year. The identical Keck II is being built 85 metres away on the peak of Mauna Kea, the Hawaiian volcano that offers astronomers one of the best viewing sites in the world. The telescopes are designed to be used both independently and in combination as an optical interferometer, which will greatly improve their joint resolving power.

According to Edward Stone, director of NASA's Jet Propulsion Laboratory and chairman of the Californian research consortium that manages Keck, it is partly the interferometry capability that makes NASA's involvement so attractive. The space agency is spending its own money to develop new techniques for optical interferometry in a test-bed project currently being carried out at Mount Palomar in California. Those techniques, to be used in the Astronomical Studies of Extrasolar Planetary Systems (ASEPS) programme, will also benefit other astronomers who are planning to use Keck.

The eventual goal of the ASEPS project is to detect Earth-sized planets around other stars, if they exist. But this is likely to require space-based instruments. The first phase of the programme, using the groundbased observations from Keck, will concentrate on indirect detection of large planets around stars that are relatively near.

The presence of planets with the mass of Uranus or Neptune can be inferred from spectral or astrometric study of a star, whose motion will be affected by the gravitational tug of a nearby massive object. ASEPS will also study protoplanetary disks - solar systems in the process of formation - which have already been observed around several

stars. Keck may also be able directly to image Jupiter-size planets around nearby stars.

An outside advisory group was due to meet in Washington this week to advise NASA on how the ASEPS programme should be run. The current plan, according to Jurgen Rahe of the agency's solar system exploration division, is to establish a core team that makes on-going observations, while funding individual investigators to conduct specific research projects in extrasolar planet detection.

\section{Bavaria bids for key role in biotech}

Munich. The southern German state of Bavaria, already known as Germany's Silicon Valley, could also become the biotechnology capital of Central Europe, despite the country's traditional hostility to such techniques, according to the state's economics minister, Otto Wiesheu.

Last week, Wiesheu announced the creation of a venture capital fund to help establish new biotechnology companies. The fund forms part of a campaign launched last July to promote new technologies in the state.

Bavaria's ministry of economics is supplying DM150 million (US\$97.35 million) to found a venture capital company for key technologies, and it wants German banks to match this amount. DM50 million will be earmarked for biotechnology.

The new money comes on top of a DM28million fund, financed almost entirely by the privatization of the Bavarian utilities

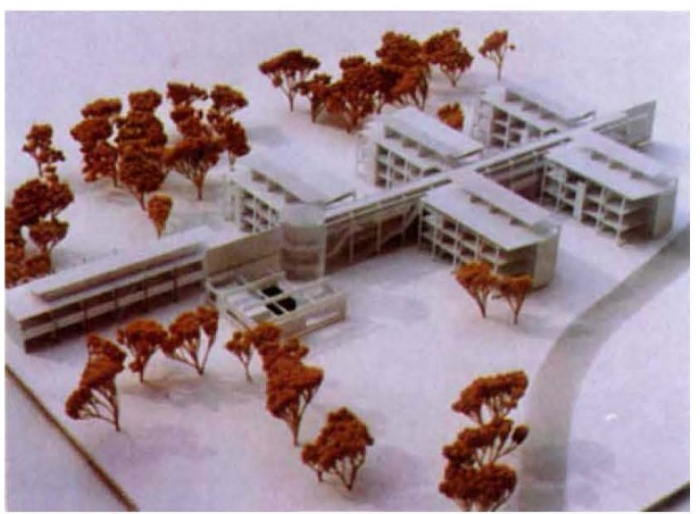

Architects' model of new Martinsried centre.

company Bayernwerk-AG, which will be used to build a large biotechnology centre in the state. DM5 million of the building fund will come from the state budget.

The programme to build up biotechnology activities in Bavaria has moved rapidly since Edmund Stoiber, the state's prime minister, announced the plan to parliament last summer. By autumn, a site in Martinsried near Munich had been chosen on which to build a 5,600-square metre 'Centre for Biotechnology', on land zation.
Until recently, the ASEPS programme was known as TOPS (Toward Other Planetary Systems). But NASA managers, worried that TOPS had become tainted when Congress cut the Search for Terrestrial Intelligence (SETI) programme from the agency's budget last year, decided to change the name. They say they are confident that the astronomical search for other planets, unlike SETI, should be safe from political attack - or at least as safe as any other project in Washington. Tony Reichhardt

donated by the Max Planck Society, Germany's principal basic research organi-

Martinsried is already a major centre for biological research, being home to two large Max Planck institutes, several university departments, and a large teaching hospital. Munich's Ludwig Maximilians University will be moving its entire chemistry and pharmacy faculties there in 1999 .

The Centre for Biotechnology will rent out laboratory and office space - initially at subsidized rates - to newly established biotechnology companies or research laboratories.

Building will start at the beginning of next year, and there will be sufficient space for at least 10 companies to move in when the first phase is completed by the end of the year. The second phase will be completed in 1996, offering space for a further 30 companies. The centre will provide major items of expensive equipment, such as sequencers and centrifuges, which companies may share without cost.

At present Germany's biotechnology sector consists of only 10 small firms. But recent amendments to Germany's tough laws on genetic engineering (see Nature 367, 210; 1994), combined with campaigns by science organizations, have improved the prospects for biotechnology.

"The anti-biotechnology movement in Germany has softened," says Axel Ullrich, head of molecular biology at the Max Planck Institute for Biochemistry at Martinsried, who was involved three years ago in setting up Sugen, a small biotechnology company jointly founded by the Max Planck Society and New York University.

"At the time we could no way think of setting it up in Germany," he says. Instead, Sugen based itself in California, but Ullrich says it is now considering installing a spinoff company in the new Martinsried centre.

Alison Abbott 